\title{
Predictors of pathological complete response to neoadjuvant chemotherapy in stage II and III breast cancer: The impact of chemotherapeutic regimen
}

\author{
IHEOMA Y. NWAOGU ${ }^{1}$, OLUWADAMILOLA M. FAYANJU ${ }^{1}$, DONNA B. JEFFE ${ }^{2,3}$ and JULIE A. MARGENTHALER ${ }^{3}$ \\ ${ }^{1}$ Department of Surgery, ${ }^{2}$ Division of General Medical Sciences, Department of Medicine, ${ }^{3}$ The Alvin J. Siteman Cancer Center, \\ Barnes-Jewish Hospital, Washington University School of Medicine, St. Louis, MO 63110, USA
}

Received May 7, 2015; Accepted June 4, 2015

DOI: $10.3892 / \mathrm{mco} .2015 .579$

\begin{abstract}
In this study, we sought to determine the predictors of pathological complete response (pCR) and compare the chemotherapeutic regimens administered to breast cancer patients with and those without $\mathrm{pCR}$. We retrospectively reviewed the data of 879 patients treated at the Alvin J. Siteman Cancer Center between 2006 and 2010, to identify patients who were diagnosed with primary stage II or III breast cancer and received neoadjuvant chemotherapy. Patients who received only neoadjuvant endocrine therapy were considered to be ineligible. Patient, tumor, and treatment characteristics, including type of chemotherapy, were compared between patients who did and those who did not achieve pCR using Chi-square or Fisher's exact tests and multivariate logistic regression analysis. Two-sided P-values of $<0.05$ were considered significant. Of the 333 patients who met the inclusion criteria, 61 (18.3\%) had documented pCR. Compared with patients not achieving pCR, a greater proportion of patients with $\mathrm{pCR}$ had stage II disease (80.3 vs. $68 \%, \mathrm{P}=0.057)$, had poorly differentiated (grade 3 ) tumors ( 82 vs. $59.2 \%, \mathrm{P}<0.001$ ), had negative lymph node involvement (41 vs. $34 \%, \mathrm{P}=0.0004$ ) and had tumors that were HER2-amplified (41 vs. 23.5\%, $\mathrm{P}=0.0054$ ). A greater proportion of patients with pCR received taxane-based chemotherapy (23 vs. $12.5 \%, \mathrm{P}=0.016$ ) or trastuzumab in conjunction with chemotherapy (41.0 vs. $16.9 \%, \mathrm{P}<0.001$ ). No patients receiving solely anthracycline-based therapy achieved pCR in our study. Our study demonstrated that, for stage II and III breast cancer, lower stage, negative lymph node involvement and HER2 receptor amplification were each associated with pCR. Taxane therapy and the concurrent use of trastuzumab were also associated with a higher likelihood of pCR.
\end{abstract}

Correspondence to: Dr Julie A. Margenthaler, The Alvin J. Siteman Cancer Center, Barnes-Jewish Hospital, Washington University School of Medicine, 660 S. Euclid Avenue, Campus Box 8109, St. Louis, MO 63110, USA

Email: margenthalerj@wudosis.wustl.edu

Key words: breast cancer, neoadjuvant chemotherapy, pathological complete response

\section{Introduction}

Neoadjuvant chemotherapy for the treatment of breast cancer has undergone significant evolution over time. Historically, neoadjuvant chemotherapy was used only for tumors considered inoperable at presentation. However, this changed after the publication of the well-known National Surgical Adjuvant Breast and Bowel Project study results, which demonstrated that neoadjuvant chemotherapy, in comparison to adjuvant, was not associated with significant differences in disease-free or overall survival and was associated with higher rates of breast-conserving surgery $(1,2)$. Neoadjuvant chemotherapy use was subsequently broadened to include large, operable tumors with the aim of achieving breast conservation.

In addition to breast conservation, neoadjuvant chemotherapy allows for an in vivo assessment of response to therapy, while also providing early treatment of the primary tumor and potential micrometastatic disease (3). Pathological complete response (pCR), often used as a surrogate endpoint to assess the efficacy of neoadjuvant chemotherapy, is also considered to be a strong prognostic measure of long-term clinical outcomes, including disease-free and overall survival (4-6). Hence, identifying and validating factors which predict $\mathrm{pCR}$ or improve pCR rates are crucial in breast cancer management.

Furthermore, anthracyclines have been considered traditionally as the most standard and active among breast cancer chemotherapy drugs. In an effort to improve pCR rates, other studies investigated the impact of combining different chemotherapeutic agents in the neoadjuvant setting (7-10). In our study, we not only sought to identify pCR rates and predictors at a single institution, but also to examine the administered chemotherapeutic regimens, and compare and contrast the regimens between patients who did and those who did not achieve pCR.

\section{Patients and methods}

Patient selection. During a retrospective review of 879 patients who were treated for a first primary breast cancer at the Alvin J. Siteman Cancer Center between January, 2006 and December, 2010, we identified patients who received neoadjuvant chemotherapy for pathologically confirmed invasive (stage II and III) breast cancer. We restricted our analysis to 
stage II and III disease in the specified 5-year period in order to evaluate a more homogeneous population of patients receiving neoadjuvant chemotherapy, with or without other neoadjuvant treatment, as neoadjuvant treatment decisions were likely made based on similar overarching guidelines during this period (based on tumor size and grade, presence or absence of lymph node metastases, receptor status and recommended chemotherapeutic regimens). The medical records of patients who received neoadjuvant chemotherapy were reviewed, to determine which of these patients' tumors exhibited a pCR, defined as no evidence of residual invasive malignancy in the breast or axilla. As ductal carcinoma in situ (DCIS) generally does not regress with chemotherapy and evidence of its impact on prognosis is equivocal $(11,12)$, patients with only residual DCIS following neoadjuvant systemic therapy were included in the pCR cohort. Patients who received only neoadjuvant endocrine therapy without mention of chemotherapy in the neoadjuvant setting were deemed ineligible for inclusion, mainly to avoid including patients who rarely achieve pCR, as previous studies have demonstrated that, while a large number of patients undergoing neoadjuvant endocrine therapy display some evidence of clinical response, pCR is infrequent (13-16). Additionally, we determined the specific neoadjuvant systemic therapy received by each of the identified patients to assess the potential impact of the chemotherapeutic regimen received on the pCR rates observed. The regimen administered to each patient was selected at the discretion of the medical oncologist, based on established standards or clinical trials in place at the time of therapy.

This study was initiated after Institutional Review Board approval was obtained, with a waiver of consent given the retrospective nature of the study.

Statistical analysis. We used the Wilcoxon rank-sum tests (for continuous variables), Chi-square or Fisher's exact tests (for categorical variables) and unadjusted logistic regression to examine clinical characteristics potentially associated with pCR, including age, race, lymph node involvement, histology, TNM stage, tumor grade, estrogen receptor (ER) and progesterone receptor (PR) status, and presence of HER2 amplification. Tumor size was not examined as a discrete variable, but rather as a component of stage. Biomarker/receptor status, stage and factors significant at $\mathrm{P}<0.2$ in unadjusted tests were included as independent variables in multivariate logistic regression models that were further refined using backwards elimination.

Two regression models for the outcome $\mathrm{pCR}$ as a yes/no binary measure were created, one featuring each individual receptor status (i.e., PR, HER2) and the other using a composite biomarker status reported of hormone receptor status plus/minus HER2 amplification. A hormone receptor-positive $(\mathrm{HR}+)$ cancer was defined as an ER-positive and/or PR-positive cancer, whereas a hormone receptor-negative (HR-) cancer was defined as an ER-negative and/or PR-negative cancer. These definitions led to the generation of four categories for comparison in the composite biomarker analysis as follows: HR+/HER2-, HR+/HER2+, HR-/HER2and HR-/HER2+. Additionally, grade was analyzed by combining well- and moderately differentiated cancers (grade 1 and 2) and comparing them to poorly differentiated cancers (grade 3 ). We report adjusted odds ratios (ORs) and $95 \%$ confidence intervals (CIs) significant at two-tailed $\mathrm{P}<0.05$. The statistical analyses were conducted using SAS 9.3 software (SAS Institute Inc., Cary, NC, USA).

\section{Results}

$p C R$ rates and predictors. Over the 5-year period reviewed, 333 patients received neoadjuvant chemotherapy. Among these patients, the majority had stage II disease (70.3\%) and tumors that were ER+ (52.3\%), PR- (55.9\%), HER2-non-amplified (HER2-; 73.3\%), poorly differentiated (i.e., grade 3; 63.4\%), and a ductal histology (77.5\%). A total of 61 patients $(18.3 \%)$ had pCR. Descriptive statistics of the study sample grouped by pCR status are shown in Table I.

In unadjusted tests, $\mathrm{pCR}$ was associated with higher tumor grade $(\mathrm{P}=0.0035)$, no lymph node involvement $(\mathrm{P}=0.0013)$, HER2 amplification $(\mathrm{P}=0.0061)$, ER-negative $(\mathrm{P}=0.027)$ and PR-negative status $(\mathrm{P}<0.001)$. Also observed was an inverse association between $\mathrm{pCR}$ and $\mathrm{HR}+/ \mathrm{HER} 2-$ status $(\mathrm{P}<0.001)$. These results are shown in Table II.

The results of separate multivariate logistic regression models, one featuring individual biomarkers and the other including composite biomarkers, are also reported in Table II. In the model featuring individual biomarkers, HER2 amplification $(\mathrm{P}=0.0095), \mathrm{PR}$-negative status $(\mathrm{P}=0.0081)$ and absence of lymph node involvement $(\mathrm{P}=0.0039)$ predicted a higher likelihood of $\mathrm{pCR}$. In the multivariate model including the composite biomarkers, the HR+/HER2- subtype and any lymph node involvement $(\mathrm{P}=0.0049)$ resulted in a lower likelihood of $\mathrm{pCR}$.

Neoadjuvant systemic therapy. Of the 333 patients in the study sample, complete records of the administered chemotherapeutic regimens were available for 323 (97\%); 252 patients received a combined regimen of anthracyclines and taxanes, 48 received a taxane-only regimen, 19 received an anthracycline-only regimen and 4 received a platinum agent-based regimen. Following completion of all the cycles of neoadjuvant chemotherapy, 46/252 patients receiving combined regimen of anthracyclines and taxanes, 14/48 patients in the taxane-only subgroup, $1 / 4$ patients receiving platinum agent-based therapy and none of the patients $(0 / 19)$ in the anthracycline-only subgroup exhibited pCR $(\mathrm{P}=0.016)$. Furthermore, administration of neoadjuvant therapy with or without trastuzumab was recorded for 329 (98.8\%) patients; 71 patients $(21.3 \%)$ received trastuzumab, either concomitant with or sequential to chemotherapy, while 258 patients did not receive trastuzumab. Following completion of all the cycles of trastuzumab treatment, 25/71 patients exhibited pCR (compared to 36/258 patients with pCR in the no-trastuzumab subgroup, $\mathrm{P}<0.001$ ). The data on neoadjuvant systemic therapy are summarized in Table III and Fig. 1.

\section{Discussion}

The results of our unadjusted analyses are in line with previously reported associations between pCR and higher tumor grade and the presence or absence of particular biomarkers. In our multivariate analysis, HER2 amplification, PR status and lack of lymph node involvement were found to be significant predictors of $\mathrm{pCR}$. 
Table I. Baseline patient and tumor characteristics.

\begin{tabular}{|c|c|c|c|c|}
\hline Characteristics & $\begin{array}{l}\text { All patients } \\
\quad(n=333)\end{array}$ & $\begin{array}{c}\text { pCR, } \\
\text { no. }(\%) \\
(\mathrm{n}=61)\end{array}$ & $\begin{array}{c}\text { No pCR, } \\
\text { no. }(\%) \\
(n=272)\end{array}$ & P-value \\
\hline Age, years [median (range)] & $48(20-83)$ & $48(27-70)$ & $48(20-83)$ & 0.8 \\
\hline Race & & & & 0.9612 \\
\hline Caucasian & $233(70.0)$ & $43(70.5)$ & $190(69.9)$ & \\
\hline African American & $93(27.9)$ & $17(27.9)$ & $76(27.9)$ & \\
\hline Other & $7(2.1)$ & $1(1.6)$ & $6(2.2)$ & \\
\hline Stage & & & & 0.0572 \\
\hline II & $234(70.3)$ & $49(80.3)$ & $185(68.0)$ & \\
\hline III & 99 (29.7) & $12(19.7)$ & $87(32.0)$ & \\
\hline Histology & & & & 0.0582 \\
\hline IDC & $258(77.5)$ & $56(91.8)$ & $202(74.3)$ & \\
\hline ILC & $22(6.6)$ & $1(1.64)$ & $21(7.7)$ & \\
\hline Mixed IDC and ILC & $20(6.0)$ & $1(1.64)$ & $19(7.0)$ & \\
\hline Inflammatory & $18(5.4)$ & $1(1.64)$ & $17(6.2)$ & \\
\hline Other & $15(4.5)$ & $2(3.28)$ & $13(4.8)$ & \\
\hline Grade & & & & 0.00065 \\
\hline 1 and 2 & $119(35.7)$ & $10(16.4)$ & $109(40.1)$ & \\
\hline 3 & $211(63.4)$ & $50(81.96)$ & $161(59.2)$ & \\
\hline Unknown & $3(0.9)$ & $1(1.64)$ & $2(0.7)$ & \\
\hline \multicolumn{5}{|l|}{ Individual biomarkers } \\
\hline Estrogen & & & & 0.026 \\
\hline $\mathrm{ER}+$ & $174(52.3)$ & $24(39.3)$ & $150(55.15)$ & \\
\hline ER- & $159(47.7)$ & $37(60.7)$ & $122(44.85)$ & \\
\hline Progesterone & & & & 0.0007 \\
\hline $\mathrm{PR}+$ & $147(44.1)$ & $15(24.6)$ & $132(48.5)$ & \\
\hline PR- & $186(55.9)$ & $46(75.4)$ & $140(51.5)$ & \\
\hline HER2 & & & & 0.0054 \\
\hline HER2+ & $89(26.7)$ & $25(41.0)$ & $64(23.5)$ & \\
\hline HER2- & $244(73.3)$ & $36(59.0)$ & $208(76.5)$ & \\
\hline Composite biomarkers & & & & 0.0007 \\
\hline HR+/HER2+ & $54(16.22)$ & $15(24.6)$ & $39(14.3)$ & \\
\hline HR-/HER2+ & $35(10.51)$ & $10(16.4)$ & $25(9.2)$ & \\
\hline HR+/HER2- & $130(39.04)$ & $10(16.4)$ & $120(44.1)$ & \\
\hline HR-/HER2- & $114(34.23)$ & $26(42.6)$ & $88(32.4)$ & \\
\hline Lymph node involvement & & & & 0.0004 \\
\hline None & $117(35.14)$ & $25(41.0)$ & $92(33.82)$ & \\
\hline Any & $100(30.03)$ & $6(9.8)$ & $94(34.56)$ & \\
\hline Unknown & $116(34.6)$ & $30(49.2)$ & $86(31.62)$ & \\
\hline
\end{tabular}

pCR, pathological complete response; IDC, invasive ductal carcinoma; ILC, invasive lobular carcinoma; ER, estrogen receptor; PR, progesterone receptor; HER2, human epidermal growth factor receptor 2.

Overexpression of HER2, a receptor-like tyrosine kinase, is shared by multiple human carcinomas; however, HER2 amplification in breast cancer is of particular significance in determining therapy and predicting outcome. Present in $20-30 \%$ of all breast cancers, HER 2 amplification potentiates growth dysregulation, oncogenesis and metastasis, all of which contribute to its association with lower disease-free and overall survival. Tumors that overexpress HER2 are also more likely to be chemoresistant, hence the importance of trastuzumab, a HER2-targeting drug, which was first approved by the Food and Drug Administration in 1998 in the management of these cancers (17). In our study, HER2 overexpression was predictive of $\mathrm{pCR}$ in the multivariate model containing individual biomarkers, thus allowing for ER, PR, and HER2 
Table II. Unadjusted and multivariate logistic regression analyses identifying independent predictors of pCR following neoadjuvant chemotherapy.

\begin{tabular}{|c|c|c|c|c|c|c|}
\hline \multirow[b]{2}{*}{ Variables } & \multicolumn{2}{|c|}{ Unadjusted analyses } & \multicolumn{2}{|c|}{$\begin{array}{c}\text { Multivariate analysis } 1 \\
\text { using composite biomarkers }\end{array}$} & \multicolumn{2}{|c|}{$\begin{array}{c}\text { Multivariate analysis } 2 \\
\text { using individual biomarkers }\end{array}$} \\
\hline & OR $(95 \% \mathrm{CI})$ & P-value & OR $(95 \% \mathrm{CI})$ & P-value & OR $(95 \% \mathrm{CI})$ & $\mathrm{P}$-value \\
\hline $\operatorname{Age}^{b}$ & $0.997(0.97-1.02)$ & 0.83 & $0.99(0.97-1.02)$ & 0.56 & $0.99(0.96-1.02)$ & 0.47 \\
\hline ER- & 1.00 (reference) & & & & 1.00 (reference) & \\
\hline $\mathrm{ER}+$ & $0.53(0.3-0.93)$ & 0.027 & - & & $1.59(0.64-3.92)$ & 0.32 \\
\hline PR- & 1.00 (reference) & & & & 1.00 (reference) & \\
\hline $\mathrm{PR}+$ & $0.35(0.18-0.65)$ & $<0.001$ & - & & $0.28(0.11-0.72)$ & 0.0081 \\
\hline HER2- & 1.00 (reference) & & & & 1.00 (reference) & \\
\hline HER 2+ & $2.26(1.26-4.04)$ & 0.0061 & - & & $2.34(1.23-4.46)$ & 0.0095 \\
\hline Grade 1 and 2 & 1.00 (reference) & & 1.00 (reference) & & 1.00 (reference) & \\
\hline Grade 3 & $3.39(1.65-6.96)$ & 0.0035 & $2.01(0.86-4.72)$ & 0.48 & $2.28(1.0-5.14)$ & 0.14 \\
\hline Stage II & 1.00 (reference) & & 1.00 (reference) & & 1.00 (reference) & \\
\hline Stage III & $0.52(0.26-1.03)$ & 0.060 & $0.60(0.27-1.32)$ & 0.096 & $0.48(0.23-1.02)$ & 0.057 \\
\hline No LNI & 1.00 (reference) & & 1.00 (reference) & & 1.00 (reference) & \\
\hline Any LNI & $0.24(0.092-0.60)$ & 0.0013 & $0.32(0.12-0.86)$ & 0.0057 & $0.28(0.11-0.79)$ & 0.0039 \\
\hline HR+/HER2+ & 1.00 (reference) & & & & 1.00 (reference) & \\
\hline HR+/HER2- & $0.21(0.078-0.55)$ & $<0.001$ & $0.27(0.11-0.67)$ & 0.0045 & - & \\
\hline HR-/HER2+ & $0.96(0.37-2.47)$ & 0.099 & $0.95(0.34-2.65)$ & 0.20 & - & \\
\hline HR-/HER2- & $0.74(0.31-1.74)$ & 0.43 & $0.59(0.26-1.33)$ & 0.84 & - & \\
\hline
\end{tabular}

${ }^{a}$ The composite biomarkers assessed in regression model were HR+/HER2+, HR+/HER2-, HR-/HER2+ and HR-/HER2-. ${ }^{b}$ Analyzed as a continuous variable. pCR, pathological complete response; OR, odds ratio; CI, confidence interval; ER, estrogen receptor; PR, progesterone receptor; HER2, human epidermal growth factor receptor 2; HR, hormone receptor; LNI, lymph node involvement.

Table III. Neoadjuvant systemic therapy received based.

\begin{tabular}{|c|c|c|c|c|}
\hline Characteristics & $\begin{array}{l}\text { All patients } \\
\quad(n=333)\end{array}$ & $\begin{array}{c}\text { pCR } \\
\text { no. }(\%) \\
(\mathrm{n}=61)\end{array}$ & $\begin{array}{c}\text { No pCR } \\
\text { no. }(\%) \\
(n=272)\end{array}$ & P-value \\
\hline Trastuzumab therapy & & & & $<0.001$ \\
\hline Yes & $71(21.3)$ & $25(41.0)$ & $46(16.9)$ & \\
\hline No & $258(77.5)$ & $36(59.0)$ & $222(81.6)$ & \\
\hline Unknown & $4(1.2)$ & $0(0.0)$ & $4(1.5)$ & \\
\hline Chemotherapy grouping & & & & 0.0161 \\
\hline Anthracyclines and taxanes & $252(75.7)$ & $46(75.4)$ & $206(75.7)$ & \\
\hline Anthracycline-based & $19(5.7)$ & $0(0.0)$ & $19(7.0)$ & \\
\hline Taxane-based & $48(14.4)$ & $14(23.0)$ & $34(12.5)$ & \\
\hline Platinum agent-based & $4(1.2)$ & $1(1.6)$ & $3(1.1)$ & \\
\hline Unknown & $10(3.0)$ & $0(0.0)$ & $10(3.7)$ & \\
\hline
\end{tabular}

pCR, pathological complete response.

statuses to be directly controlled for in relation to one another. In the model with composite biomarkers, we also observed that the ER+/PR+/HER2- subtype was negatively associated with $\mathrm{pCR}$. The aggregate of these findings suggests that there may be a complex interplay between the tumor biology and treatment with trastuzumab. In reference to pCR rates, we are currently unable to disentangle the potential biological effects of HER2 amplification from the potential benefits gained from trastuzumab treatment. Additional prospective studies are required to elucidate this matter.

Lack of lymph node involvement was also found to be a significant predictor of $\mathrm{pCR}$ in patients with HER 2 amplification 


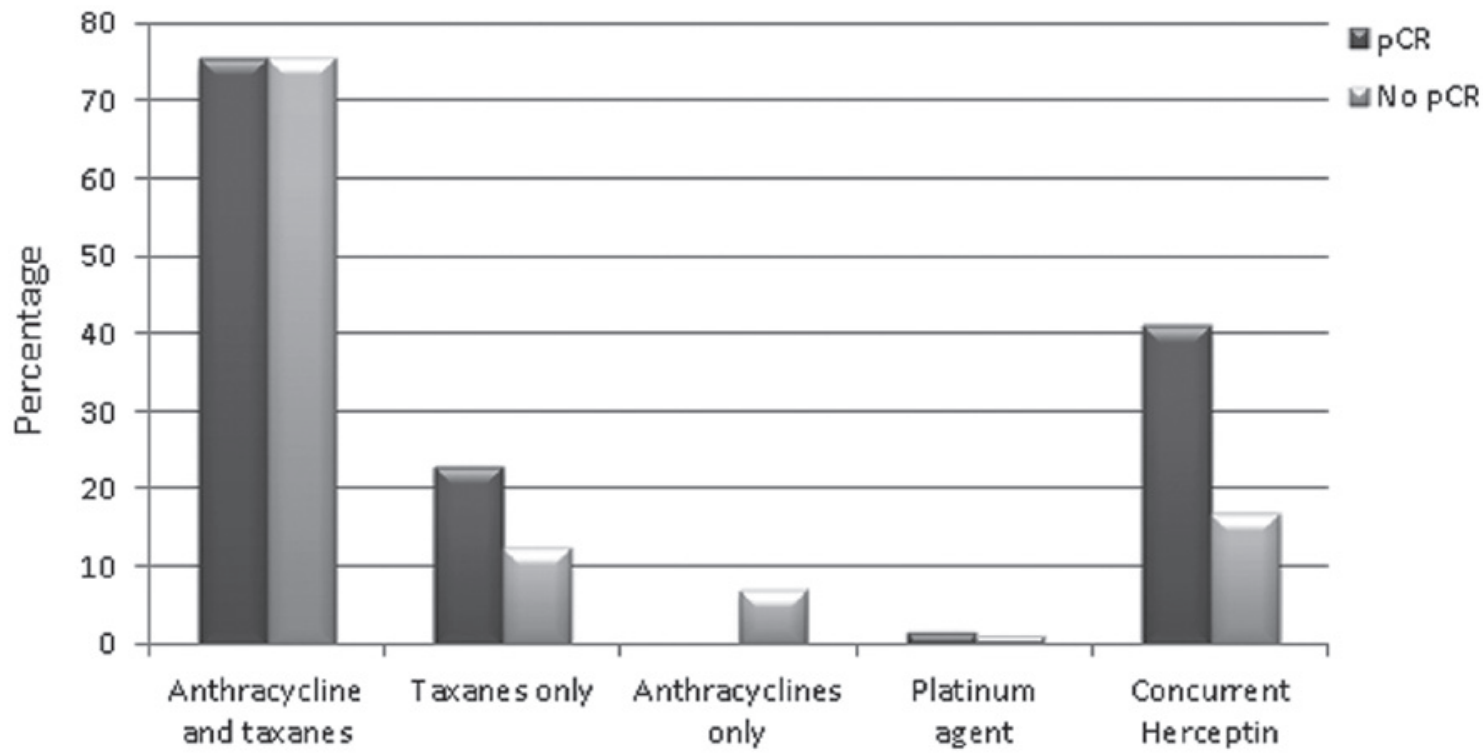

Figure 1. Chemotherapeutic regimen used in patients with or without pathological complete response (pCR).

without ER or PR expression, even after adjusting for stage in the regression model. Lymph node involvement at initial presentation is a well-established outcome prognosticator in breast cancer and patients with breast pCR but residual nodal disease have lower rates of overall survival compared with patients who experience breast as well as nodal pCR $(1,18)$.

The assessment of the type of neoadjuvant systemic therapy received, highlights the fact that the majority of the treated patients at our institution received the standard recommended regimen, while also exhibiting improved pCR rates when taxanes are used or added to anthracycline therapy. Multiple trials evaluating a broad range of chemotherapy drugs have also demonstrated that the use of anthracyclines in combination with taxanes is associated with improved pCR rates $(7,10)$. Hence, the consensus statement of the St. Gallen Conference, states that a standard neoadjuvant chemotherapy regimen should include both an anthracycline and a taxane (19).

Platinum agents are DNA-damaging agents, which have been found to be particularly beneficial in improving pCR rates in triple-negative breast cancer (TNBC), particularly BRCA-mutated tumors (which represent up to $50 \%$ of all TNBCs). In a meta-analysis of 28 studies, the pooled pCR rate noted following addition of a platinum agent to a standard neoadjuvant chemotherapy regimen for TNBC was $45 \%$ (20). Another single-institution study reported pCR rates of $22 \%$ following neoadjuvant treatment with single-agent cisplatin therapy in patients with stage II or III TNBC (21). In our study, all 4 patients receiving platinum agents had TNBC and they received single-agent therapy with the platinum agent. Of note, the sole patient exhibiting pCR following administration of the platinum agent had a higher tumor grade (grade 3), lower stage (stage II) and no lymph node involvement. To the best of our knowledge, a review of the literature has retrieved no data to suggest that the improvement in pCR rates following addition of platinum agents to standard neoadjuvant chemotherapy in TNBC translates to improvements in overall survival or disease-free survival. More studies are required to evaluate the potential survival benefits of adding platinum-based therapy to standard neoadjuvant chemotherapy for patients with TNBC.

Our study had certain limitations. First, we were unable to establish causation, but could only show association, due to the fact that retrospective studies are prone to patient and treatment selection bias. Additionally, potential confounding variables may not always be recognized or recorded, due to a lack of knowledge regarding how they interact with the outcome of interest. Second, we were unable to control for administration of trastuzumab therapy with regard to the association of HER2 amplification with pCR. As the provision of HER2-targeted therapy has recently become more standardized, future prospective studies will undoubtedly be better equipped to investigate this issue. Third, all the patients in our study were treated at a high-volume, NCI-designated comprehensive cancer center, although the majority of the patients in this country obtain their chemotherapy from non-academic, community medical oncologists. This may translate to potential differences in patient population, treatment received, treatment duration, or even the definition of pCR; thus, the extent to which our patients exhibit $\mathrm{pCR}$ and the reasons why they do so, may not reflect the experience of breast cancer patients receiving care outside a comprehensive cancer center. Finally, details regarding the optimal chemotherapy dose, treatment duration, or concurrent vs. sequential administration for improving pCR rates were not obtained in this study. Other studies are currently underway, however, to address optimal administration times in the neoadjuvant setting to improve pCR rates.

In conclusion, we found in the regression model using the composite HR variable that HR+/HER2- tumors were significantly less likely to undergo pCR compared with HR+/HER2+ tumors, and that tumors with any lymph node involvement were significantly less likely to undergo pCR compared with tumors without lymph node involvement. Additionally, we found that the pCR rates were higher among patients receiving trastuzumab or taxane therapy in addition to anthracycline therapy. 


\section{Acknowledgements}

The study of Dr Nwaogu was supported by Dr Graham Colditz and Dr Timothy J. Eberlein. Dr Fayanju was supported by the NIH Ruth L. Kirschstein National Research Service Award Institutional Research Training grant (no. 5T32CA009621-24). Dr Jeffe was supported in part by the NCI Cancer Center support grant (no. P30 CA091842) to the Alvin J. Siteman Cancer Center at Barnes-Jewish Hospital and Washington University School of Medicine, St. Louis, MO, USA. Portions of this study's findings were presented at the American Society of Breast Surgeons 15th Annual Meeting, April 30-May 4, 2014.

\section{References}

1. Fisher B, Bryant J, Wolmark N, et al: Effect of preoperative chemotherapy on the outcome of women with operable breast cancer. J Clin Oncol 16: 2672-2685, 1998.

2. Wolmark N, Wang J, Mamounas E, Bryant J and Fisher B: Preoperative chemotherapy in patients with operable breast cancer: Nine-year results from National Surgical Adjuvant Breast and Bowel Project B-18. J Natl Cancer Inst Monogr 30: 96-102, 2001.

3. Van der Hage JA, van de Velde CJ, Julien JP, Tubiana-Hulin M, Vandervelden C and Duchateau L: Preoperative chemotherapy in primary operable breast cancer: Results from the European Organization for Research and Treatment of Cancer trial 10902. J Clin Oncol 19: 4224-4237, 2001.

4. Kong X, Moran MS, Zhang N,Haffty B and Yang Q: Meta-analysis confirms achieving pathological complete response after neoadjuvant chemotherapy predicts favourable prognosis for breast cancer patients. Eur J Cancer 47: 2084-2090, 2011.

5. Rastogi P, Anderson SJ, Bear HD, et al: Preoperative chemotherapy: Updates of National Surgical Adjuvant Breast and Bowel Project Protocols B-18 and B-27. J Clin Oncol 26: 778-785, 2008.

6. Di Cosimo S, Arpino G and Generali D: Neoadjuvant treatment of HER 2 and hormone-receptor positive breast cancer - moving beyond pathological complete response. Breast 23: 188-192, 2014.

7. Hirano A, Shimizu T, Imamura H, et al: The combination of epirubicin plus docetaxel as neoadjuvant chemotherapy in locally-advanced breast cancer. Anticancer Res 26 (1b): 581-584, 2006.

8. Gianni L, Eiermann W, Semiglazov V, et al: Neoadjuvant and adjuvant trastuzumab in patients with HER2-positive locally advanced breast cancer (NOAH): Follow-up of a randomised controlled superiority trial with a parallel HER2-negative cohort. Lancet Oncol 15: 640-647, 2014.

9. McCarthy N, Boyle F, Zdenkowski N, et al; Australia and New Zealand Breast Cancer Trials Group: Neoadjuvant chemotherapy with sequential anthracycline-docetaxel with gemcitabine for large operable or locally advanced breast cancer: ANZ 0502 (NeoGem). Breast 23: 142-151, 2014.
10. Bear HD, Anderson S, Smith RE, et al: Sequential preoperative or postoperative docetaxel added to preoperative doxorubicin plus cyclophosphamide for operable breast cancer: National Surgical Adjuvant Breast and Bowel Project Protocol B-27. J Clin Oncol 24: 2019-2027, 2006.

11. Mazouni C, Peintinger F, Wan-Kau S, et al: Residual ductal carcinoma in situ in patients with complete eradication of invasive breast cancer after neoadjuvant chemotherapy does not adversely affect patient outcome. J Clin Oncol 25: 2650-2655, 2007.

12. Von Minckwitz G, Untch M, Blohmer JU, et al: Definition and impact of pathologic complete response on prognosis after neoadjuvant chemotherapy in various intrinsic breast cancer subtypes. J Clin Oncol 30: 1796-1804, 2012.

13. Cataliotti L, Buzdar AU, Noguchi S, et al: Comparison of anastrozole versus tamoxifen as preoperative therapy in postmenopausal women with hormone receptor-positive breast cancer: The pre-operative 'Arimidex' compared to tamoxifen (PROACT) trial. Cancer 106: 2095-2103, 2006.

14. Semiglazov VF, Semiglazov VV, Dashyan GA, et al: Phase 2 randomized trial of primary endocrine therapy versus chemotherapy in postmenopausal patients with estrogen receptor-positive breast cancer. Cancer 110: 244-254, 2007.

15. Ellis MJ, Suman VJ, Hoog J, et al: Randomized phase II neoadjuvant comparison between letrozole, anastrozole, and exemestane for postmenopausal women with estrogen receptor-rich stage 2 to 3 breast cancer: Clinical and biomarker outcomes and predictive value of the baseline PAM50-based intrinsic subtype - ACOSOG Z1031. J Clin Oncol 29: 2342-2349, 2011.

16. Tan MC, Al Mushawah F, Gao F, et al: Predictors of complete pathological response after neoadjuvant systemic therapy for breast cancer. Am J Surg 198: 520-525, 2009.

17. Harari D and Yarden Y: Molecular mechanisms underlying ErbB2/HER2 action in breast cancer. Oncogene 19: 6102-6114, 2000.

18. McCready DR, Hortobagyi GN, Kau SW, et al: The prognostic significance of lymph node metastases after preoperative chemotherapy for locally advanced breast cancer. Arch Surg 124: 21-25, 1989.

19. Goldhirsch A, Wood WC, Coates AS, Gelber RD, Thürlimann B and Senn HJ; Panel members: Strategies for subtypes - dealing with the diversity of breast cancer: Highlights of the St. Gallen International Expert Consensus on the Primary Therapy of Early Breast Cancer 2011. Ann Oncol 22: 1736-1747, 2011.

20. Petrelli F, Coinu A, Borgonovo K, Cabiddu M, Ghilardi M, Lonati $\mathrm{V}$ and Barni S: The value of platinum agents as neoadjuvant chemotherapy in triple-negative breast cancers: A systematic review and meta-analysis. Breast Cancer Res Treat 144: 223-232, 2014.

21. Silver DP, Richardson AL, Eklund AC, et al: Efficacy of neoadjuvant cisplatin in triple-negative breast cancer. J Clin Oncol 28: 1145-1153, 2010. 